\title{
Néolithisation en Afrique du Nord : Introduction
}

\section{Colette Roubet}

\section{OpenEdition}

Journals

Édition électronique

URL : https://journals.openedition.org/encyclopedieberbere/2715

DOI : 10.4000/encyclopedieberbere.2715

ISSN : 2262-7197

\section{Éditeur}

Peeters Publishers

\section{Édition imprimée}

Date de publication : 5 octobre 2012

Pagination : 5444-5461

ISBN : 978-90-429-2718-6

ISSN : 1015-7344

\section{Référence électronique}

Colette Roubet, « Néolithisation en Afrique du Nord : Introduction », Encyclopédie berbère [En ligne], 34 | 2012, document N40, mis en ligne le 15 décembre 2020, consulté le 17 février 2022. URL : http:// journals.openedition.org/encyclopedieberbere/2715; DOI : https://doi.org/10.4000/ encyclopedieberbere. 2715

Ce document a été généré automatiquement le 17 février 2022.

(c) Tous droits réservés 


\title{
Néolithisation en Afrique du Nord: Introduction
}

\author{
Colette Roubet
}

1 Sur le vaste espace africain septentrional, au contour méditerranéen, saharien et atlantique, plusieurs études permettent d'accéder à des informations sur les étapes de transition vers la Néolithisation et celles de son déroulement. Cette transition s'accompagne de changements socio-économiques; elle peut s'appréhender en réunissant des données paléo-environnementales et climatiques sur le Pléistocène terminal (Paléolithique) et l'Holocène. Mais c'est en contextualisant les données culturelles que l'orientation du concept de Néolithisation cible mieux la complexité et l'impact de mutations comportementales qui installent une économie de production. Cette conception européenne et proche-orientale est-elle partout applicable sur ce territoire (Garcea 2004) ? Une africanisation de la néolithisation se dessine déjà (Huysecom 1996, 2006, 2009). Cet auteur suggère de redonner à la céramique un statut culturel et économique spécial compte tenu de son ancienneté en Afrique ( $\mathrm{X}^{\mathrm{e}}$ millénaire) comme pour mieux souligner une maîtrise des prédations végétales ciblées dans l'environnement. Pour les territoires sous influence méditerranéenne et atlantique, les conditions d'un affranchissement des contraintes d'une prédation exclusive de la subsistance se précisent en certains endroits. Pour les territoires sahariens, des périodes de péjorations climatiques durant l'Holocène limitent la portée des résultats, montrent des adaptations aux ressources (quête de milieux aquatiques), même décroissantes, voire refugiées, pour créer avec ingéniosité des « oasis »- espaces originaux et privilégiés. Une démarche scientifique peut prendre en compte tous les cas rencontrés, y compris ceux concernés par les effets de seuil anthropique induits par une désertification en développement, migrant vers le nord depuis les basses latitudes et altitudes.

2 Cette démarche consiste à essayer de comprendre comment au début de l'Holocène, l'Homme, chasseur-collecteur-pêcheur, placé dans des conditions d'instabilité climatique et de pression démographique (facteur de dynamisme), affectant les biotopes et leurs ressources naturelles, put, ou non, maîtriser ses sources de nourriture, 
en pratiquant d'abondantes collectes de végétaux de façon récurrente et sélective et en assujettissant des espèces animales et végétales, ou en les adoptant déjà domestiquées, enclenchant, ou non, une économie de production.

Cet objectif retenu en Libye par C.-B. McBurney fut testé en Cyrénaïque pour montrer le passage de la chasse à la domestication animale par E. Higgs dans l'abri d'Haua Fteah en 1967. Il fut adopté et mis en place en Algérie par C. Roubet avec E. Higgs et P. Carter en 1979, dans la grotte de Capéletti (Aurès). Mais il ne s'imposa pas au nord de l'Afrique, parce que l'Ecole de Préhistoire d'Alger crut longtemps devoir privilégier le contenu culturel et cognitif d'un contexte archéologique, conservatoire d'innovations techniques et symboliques, pour n'accorder qu'une importance secondaire aux témoins alimentaires, souvent absents ou mal conservés, se privant alors des données essentielles d'une réflexion sur les comportements.

Nous allons montrer comment articuler dans le cas de sites stratifiés holocènes, des séries d'informations croisées: les unes tirées de la caractérisation des conditions chrono-sédimentaires et climato-environnementales locales et régionales, les autres issues de contenus culturels et de comportements sociaux. La mise en synergie de ces informations devrait aboutir à proposer de nouvelles stratégies socio-économiques en observant :

- Un changement dans la composition de la subsistance, soulignant une complémentarité des ressources issues d'une acquisition par production et prédation, animales, végétales et autres, permise par la mise en place d'un système d'activités intégrées. Certaines, impliquant l'élevage d'animaux domestiques et une mise en culture de certains végétaux, ont pour corollaire une mobilité contingente et saisonnière, à l'origine des transhumances, du « nomadisme* » et/ou une semi-sédentarisation,

- Un changement dans le contenu culturel, matériel et immatériel soulignant des modifications et innovations dans l'équipement en relation avec des stratégies domestiques et symboliques, relationnelles et sociétales (échanges), greffées sur un ensemble de traditions antérieures.

5 C'est à terme la transmission de ces comportements fondamentaux qui est à l'origine de la constitution d'une identité comportementale berbère, méditerranéenne et africaine.

Pour harmoniser nos procédures à l'intérieur de l'espace défini, il importe d'identifier les causes extérieures et naturelles des changements puis les réactions anthropiques. Or c'est par un long processus de mutations mal identifiées, non linéaires, localement interrompues, que sont passées des communautés territorialisées de chasseurscueilleurs-pêcheurs. Pour en rendre compte, il conviendra d'admettre le principe de complémentarité de ressources fluctuantes, révélateur d'une spécialisation climatorégionale des comportements. Il importe donc de continuer à dresser une cartographie chronologique régionale de ces communautés, de n'en marginaliser aucune, de préciser leur statut économique, avant d'assembler un nouveau puzzle pré-néolithique. En s'appuyant aussi sur chaque fait exogène, insolite, rare, et en l'articulant aux déplacements, on parvient à mailler et valoriser des relations ciblées, à l'origine d'« un vivre ensemble », fondement antérieur à cette néolithisation (Roubet et al. 2011).

7 Dans la présentation des notices sur le Néolithique ou la Néolithisation, selon l'état d'avancement des travaux, nous apporterons des résultats récents obtenus au Maroc (Ballouche, Ouchaou, El Idrissi), en Algérie tellienne (Roubet, Kherbouche) et atlasique (Roubet), en Tunisie (Aouadi, Dridi), en Libye (de Faucamberge, Barich). 


\section{Modifications climato-territoriales}

8 La première partie de l'Holocène, qui voit se mettre en place les étapes initiales de la néolithisation en Afrique du Nord, est marquée par des changements territoriaux et environnementaux consécutifs aux changements climatiques post-glaciaires. Pour les sociétés, ce sont bien moins les changements climatiques en eux-mêmes qui ont un sens que leurs impacts sur les espaces et les milieux.

9 Après la remontée du niveau des mers qui redessina les côtes, on vit s'isoler des îles et s'immerger des plaines côtières, pourvoyeuses durant le Pléistocène final d'une nourriture halieutique très exploitée par les Cro-Magnons nord-africains. Cet événement climato-territorial paraît avoir tant restreint le littoral qu'il pourrait expliquer la migration méridionale des Hommes de Mechta-Afalou (vers le sud-ouest: région de Tiaret et Columnata; vers le sud-est: Bou-Saada et El Hamel). Ailleurs, plusieurs fluctuations climatiques (humide, aride) affectèrent les paysages nordafricains.

10 Sur le pourtour méditerranéen, certains grands traits d'une évolution climatique permettent d'identifier trois phases, aux limites fluctuantes selon les auteurs: une première phase humide dès le début et durant l'Holocène ancien (11 500 ? - 8200/7000 cal BP), une seconde phase de l'Holocène moyen un peu moins humide (8200/7000 ? $5500 / 5000$ cal BP) et une troisième phase devenant de plus en plus sèche au cours de l'Holocène récent (5500/5000 cal BP? - Actuel) marquée par une aridification progressive. Cette longue histoire climatique, loin d'être linéaire, a traversé plusieurs épisodes remarquables dont un événement majeur autour de 8200 cal BP.

11 Durant l'Holocène ancien en Afrique du Nord, l'installation des végétations et des faunes méditerranéennes typiques, marque une reconquête forestière après le Younger Dryas (Salamani 1991; Ballouche 2001; Benslama et al. 2010) mettant en place, en altitude, des formations forestières dites climaciques: chênaies caducifoliées et sclérophylles, entre autres, dans l'étage méso-méditerranéen des plaines et des moyennes montagnes. La présence de certaines d'entre elles très particulières, comme les cédraies, souligne l'originalité des massifs du Rif* au Maroc septentrional, des Babors* et de l'Aurès* en Algérie, durant cet humide. En basse altitude aussi, dans les régions aujourd'hui semi-arides du Maroc oriental ou du sud tunisien, on a pu constater une diversification plus grande dans la composition des steppes. Sur le plan hydrologique, cette période a été marquée par de nombreux témoignages de hauts niveaux lacustres dans des régions arides comme l'est devenu le Grand Erg occidental du Sahara algérien. Ces hauts niveaux se sont développés dès 10500 ans cal BP, avec un maximum entre 8800 et 7350 ans cal BP, et une décroissance que l'on peut suivre jusqu'à l'assèchement final vers 5000 ans cal BP (Callot et Fontugne 2008).

12 Cependant, c'est aussi lors de cet Holocène récent humide en Afrique du Nord que de nombreux auteurs identifient un bref épisode abrupt, froid et sec, centré sur $8200 \mathrm{cal}$ $\mathrm{BP}$, pouvant être reconnu entre 8300 et $8000 \mathrm{cal} \mathrm{BP}$ dans tout l'hémisphère nord, $\mathrm{y}$ compris le bassin méditerranéen et le Sahara (Alley et al. 1997; Reimer et al. 2004 ; Weninger et al. 2006; Berger et Guilaine 2009). On peut rattacher à cet épisode différents événements signalés dans des travaux de paléoclimatologie et de stratigraphie préhistorique en Algérie, notamment vers Ouargla* (Gasse et al. 1987), en Libye tassilienne (Petit-Maire et al. 1979; Cremaschi et Di Lernia 1998, 1999) (Tableau 1, Maley 1981, 2010) et dans le Bassin du Tchad (fig. 1). 
Tableau 1. Représentation synthétique de la succession des phases arides et humides au Sahara depuis 22000 ans. La chronologie est présentée à l'aide de datations calendaires à gauche (calibration d'après Reimer et al 2004) et avec des datations au radiocarbone $14 \mathrm{C}(\mathrm{BP})$ à droite. D'après J. Maley 2010, p. 243.

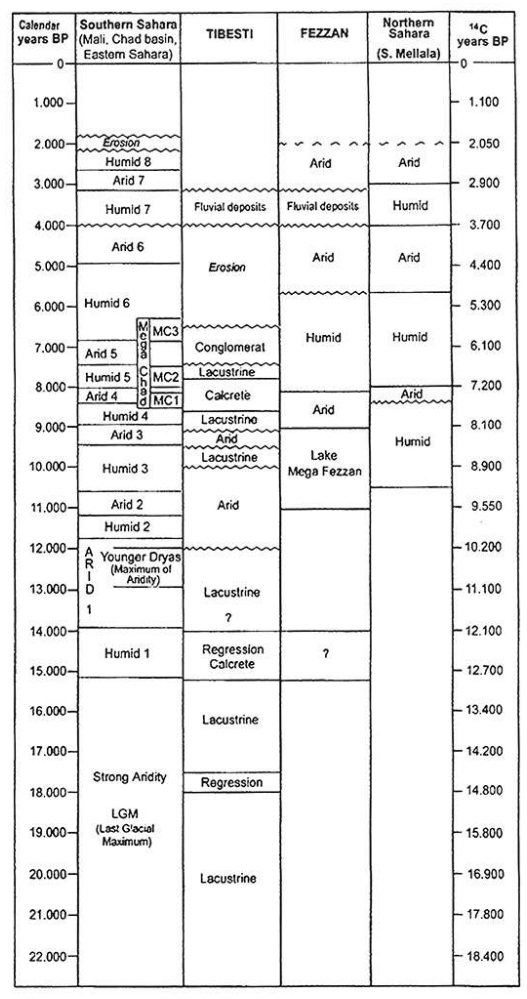

Erosion, main phase $2.400-1.900 \mathrm{BP}$

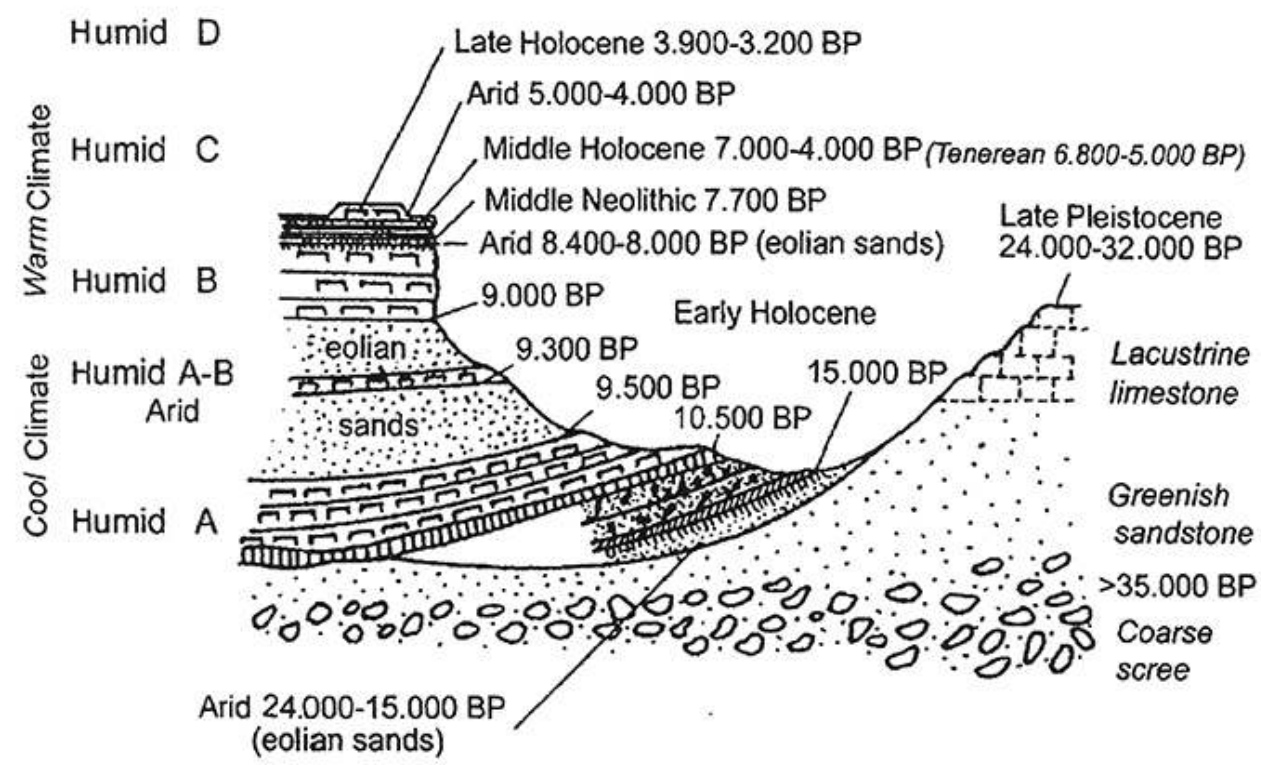

Fig. 1. Dépôts récents du Quaternaire près de Fachi, dépression de Dogomboulo (Bassin oriental du fleuve Niger) au Tchad. L'Holocène est subdivisé en six Membres (A-E). Les datations sont calibrées (Reimer et al. 2004). On distingue nettement l'épisode climatique aride à 8200 calBP apparu après le grand humide (B). D'après J. Maley 2010, p. 237. 

plus humides qu'au début de l'Holocène (Hutterer et al. 2011). Ces conditions climatoenvironnementales favorables semblent s'être généralement maintenues jusque vers 5500 cal. BP, avec une dégradation parfois identifiée entre 6500 et 6000 cal BP (Zielhoffer \& Linstädter 2006).

Si l'Holocène ancien-moyen comprend deux périodes assez humides au Sahara comme au Maghreb, l'Holocène supérieur-récent, d'abord tempéré, est devenu à partir de 5500/5000 cal BP, selon les altitudes et les régions, progressivement plus sec jusqu'à ce que l'aridité s'installe définitivement.

15 A des échelles sous-régionales ou locales, ces conditions générales paléoenvironnementales pourraient avoir été plus contrastées, en raison d'un espace nordafricain aux reliefs fortement compartimentés, mais aussi pour de multiples causes microclimatiques, édaphiques, etc., ayant pu entraîner des bilans hydriques variés et aléatoires.

16 Ainsi, par exemple, dans le nord-est marocain, Hutterer et al. (2011) mettent en évidence, à partir de données quantitatives fournies par les gastéropodes terrestres, un environnement assez sec entre $9700 \mathrm{cal}$ BP et 8800 cal BP. Un autre exemple est fourni par différents dépôts lacustres du Grand Erg occidental, dont le caractère salé, ou d'eau douce, loin d'avoir une signification paléoclimatique, résulterait plutôt d'un jeu d'équilibre, latitudinal, entre la nappe locale d'eau douce du Grand Erg et une paléonappe salée de la hamada au nord (Callot \& Fontugne 2008). Enfin, l'épisode froid et sec généralisé vers 8200 cal BP ne semble pas avoir été enregistré dans les dépôts du fond des vallées ni dans le fonctionnement hydrologique de la Moulouya et du Kert au Maroc ou de la Medjerda en Tunisie (Zielhoffer et al. 2008).

La question des rapports des peuplements préhistoriques aux changements climatiques reste posée, avec plus ou moins de pertinence. Dans le Bassin Méditerranéen oriental, pour Berger \& Guilaine (2009), l'événement à 8200 cal BP serait parfaitement synchrone de ruptures culturelles, signalant la transition en Europe du Mésolithique au Néolithique ; il a été observé dans le remplissage de nombreuses grottes depuis la Grèce jusqu'à la péninsule Ibérique, entre 8500 et 8000 cal BP.

18 Un tel épisode pourrait avoir eu en Afrique du Nord aussi de réelles conséquences, mais celles-ci sont encore mal mesurées (Rahmani 2004; Zielhoffer et al. 2008). Sur les Hautes-Plaines du Maghreb oriental, on pourrait en percevoir les effets dans le développement soudain et organisé d'activités de pêche, près des lagunes du Columnatien* et de l'Elassolithique* s'accompagnant de l'adoption de nouveaux comportements techniques (ultra-microlithisme) (Roubet, EB XXXVIII-XXXIX, p. 4271-4282). D'autres catégories de prédations des Capsiens durant l'Holocène ancien suggèreraient aussi un changement de subsistance en région steppique (Némencha*). L'hypothèse avancée à partir de certains témoins fauniques de restes alimentaires (nature, dimensions) repose sur des modifications environnementales ayant pu entraîner une raréfaction du pâturage et le départ des grands herbivores, conduisant à la capture de petites espèces et des collectes de gastéropodes terrestres (Jackes et Lubell 2008). En revanche, pour Zielhoffer et al. (2008), le début du Néolithique en Méditerranée Occidentale ne semble pas devoir être relié à des changements climatiques ou environnementaux identifiés. Ce serait plutôt à la suite de processus 
culturels, économiques et sociaux, induits par des phénomènes migratoires et d'acculturation, que se serait opérée la transition Épipaléolithique/Néolithique.

Bien qu'insuffisante et provisoire, cette esquisse chrono-climatique installe dans ce territoire nord-africain une succession chronologique alternée de plusieurs épisodes humides et arides durant l'Holocène, aux répercussions différentes selon les latitudes et l'altitude. Etablir des liens, s'ils ont existé, entre ces changements climatiques et l'adoption ou non de nouveaux comportements, restera un objectif à poursuivre à travers des programmes pluridisciplinaire intégrés, mais sans déterminisme excessif, à condition qu'il s'avère opportun et non simplificateur. Sachant qu'en territoire saharien, aussi bien en Egypte qu'en Libye tassilienne, des occupations pastorales synchrones d'épisodes humides de l'Holocène ancien ont été reconnues, et se sont encore maintenues jusqu'au IV ${ }^{e}$ millénaire cal BP dans certains secteurs seulement, prolongeant ainsi le processus pastoral saharien.

\section{Modifications et adaptations comportementales}

\section{Le cas de l'Egypte}

Pour l'Egypte, c'est loin du Nil, dans le désert occidental, que les données du début de l'Holocène (fauniques, culturelles et sociales), issues de plusieurs fouilles, ont établi des rythmes de fréquentations (présence/absence) alternées, dépendantes de l'eau, ressource majeure d'un vivre social, rappelée ici, après J. Sutton et d'autres auteurs: «Le società che si affermano nel Sahara durante la fase piu umida dell'olocene mostrano uno spiccato legame con l'acqua, che appare il principale fattore condizionante il viveresociale " (Barich 2010, p. 206). Les séquences chrono-stratigraphiques des sites de Bir Kiseiba et Nabta Playa (Wendorf, Schild \& Close 1984 ; Wendorf, Schild \& Associates 2001), ont laissé A. Gautier envisager (1984), que pour compenser la capture de nombreux petits mammifères durant la première pulsation humide entre 10000 et 9500 cal BP, (Tableau 1) se développa une «incipient domestication» de quelques bovins de grande taille.

\section{Le cas de la Libye tassilienne}

21 Pour la Libye tassilienne, la faune de la Tadrart Acacus étudiée par A. Gautier (1987) le conduisit à admettre aussi cette hypothèse, non confirmée depuis (in litt. B. Barich 30/04/2010). A Wadi Ti-n-Torha, Abri-Est, les ossements de quelques bovins sont datés entre :

$8540 \pm 140$ BP soit $9530 \pm 140$ cal BP ou $7580 \pm 140$ cal BC et $7990 \pm 070$ BP soit $8925 \pm 200$ cal BP ou $6975+200$ cal BC.

Dans l'Edeyen de Murzuk*, Mutri, Di Lernia et Garcea (EB XXXII, p. 5109-5112) ont établi une séquence détaillée débutant par un "Acacus ancien » sans faune domestique, suivi d'une phase aride, puis d'une phase pastorale « Acacus récent ». Trois phases ensuite s'échelonneraient (Barich 2010, p. 214) :

- Un « Pastoral ancien ", avec bovins domestiques, établi à Uan Muhuggiag* (Barich, EB XXXII, p. 5101-5104) et daté entre : 7400 et 6400 BP soit 8200-7300 cal BP ou 6250-5350 cal BC, avec un niveau de base de : 
$7438 \pm 220$ BP soit $8250 \pm 220$ cal BP ou $6300 \pm 220$ cal BC. Cet épisode bénéficia à Murzuk* de beaucoup d'humidité favorisant des installations alternées en montagne et au bord des lagunes. présence en région sub-désertique, mais au bord de lagunes, de quelques mammifères durant le premier épisode humide, alors que les chasses ne procuraient que gazelles et lièvres. D'autres considérations sur l'assujettissement des bovins (Barich 2010) admettent l'établissement d'un rapport particulier au début du processus de domestication. Celui-ci résulterait d'une longue expérience éthologique, sans lien direct avec une consommation de viande, d'ailleurs peu abondante, ayant conduit, par le biais de pâturages disponibles, à apprivoiser cet animal attachant, accessible (lait, sang ; cf. pour des périodes récentes, Le Quellec 2011) et mobile. Ce processus diffère de celui du Proche-Orient, établi à partir d'un autre rapport liant l'homme à l'animal domestique, au sol et aux plantes cultivées et récoltées (chaumes-pâturage), impliquant une sédentarisation, au moins saisonnière. Au Sahara, les sources documentaires concernant des périodes sub-actuelles signalent en période de pluies abondantes le retour d'une biodiversité (plantes animaux) près et dans les guelta, favorable aux piégeages, aux sélections et collectes, nourriture stockée pour une consommation différée (Gast 1968).

\section{Le cas de La Libye-Cyrénaïque, Algérie, Tunisie, Maroc}

On observe que la néolithisation débute en région septentrionale par une phase plutôt pastorale, de la Cyrénaïque au Maroc, contemporaine de l'Holocène ancien-moyen, n'ayant pas été précédée par une domestication animale initiale. En considérant les entités géographiques concernées, il devient évident que ce sont les territoires karstiques qui ont bien conservé les bio-documents des occupations pastorales.

On remarque en région atlasique que les premiers groupes humains qui s'aventurent à l'intérieur de massifs sont des pasteurs qui s'installent en grottes et sous abris. Ils s'implantent en altitude et créent de vastes « territoires à vocation pastorale » (Tunisie centrale : cas probable de Doukanet el-Khoutifa (?) ; Némencha* : cas du Damous elAhmar*; Aurès* : cas de Capéletti*; chaînons occidentaux sahariens algériens).

En zone tellienne méditerranéenne, bien que ce comportement n'ait pas encore été étudié, il semble possible de reconnaitre aux lieux attractifs choisis en montagne par les bergers une adéquation certaine avec leurs préoccupations pastorales. Les données de la subsistance révèlent une consommation d'animaux domestiques en Libye, Cyrénaïque (cas d'Haua Fteah et d'Abou Tamsa*); en Tunisie dans les MogodsKroumirie (grotte du Kef el-Agab*), en Algérie, dans les Babors* (secteur occidental) 
(cas de GLD1 de l'Adrar Gueldaman*) et au Maroc, dans le Rif* pastoral néolithisé dès le $\mathrm{VI}^{\mathrm{e}}$ millénaire cal BC (grotte de Kaf Taht el-Ghar, d'Ifri n Ouadadane et d'Ifri Armas).

31 Tandis que dans le Bas Sahara algérien (Aumassip 2001), alors que quelques gisements stratifiés de l'Holocène ancien et moyen ont été étudiés (Hassi Mouillah*, Bordj Melalla), certains apparaissent désormais en surface, tous subissent encore une telle déflation qu'aucun n'a conservé de restes osseux identifiables. L'absence de biodocuments constitue-t-elle une réponse négative au processus de production de subsistance ? Ce n'est pas ce que pense G. Aumassip.

Les informations actuellement réunies autorisent l'hypothèse d'une néolithisation pastorale très localisée, enclenchée par acculturation et acquisition de nouveaux savoir-faire, pouvant venir du nord-ouest méditerranéen et d'Espagne (via le Maroc), du sud par le Niger (?) et de l'est par l'Egypte (Libye, Tunisie, Algérie). Les données fauniques sur la domestication animale font état de cinq espèces domestiques identifiées au Maroc (mouton, chèvre, bovin, porc et chien : cf. Ouchaou, N48), trois en Algérie (mouton, chèvre, bovin ; Roubet, N45 \& 46), deux en Tunisie (mouton et chèvre ; Aouadi \& Dridi, N44) et deux aussi en Cyrénaïque (mouton et chèvre ; cf. Barich, N42 ; de Faucamberge, N43). On peut espérer disposer pour les gisements du Maroc de données sur la microfaune commensale, liée aux occupations holocènes et aux céréales.

\section{Le domaine végétal durant l'Holocène : la domestication des plantes}

33 La présence de céréales domestiques a été établie au Maroc et en Libye méditerranéenne. Le site de Kaf Taht el-Ghar (Tanger, Maroc) a livré des témoins polliniques de type céréales et des grains de blés domestiques carbonisés, représentés par Triticum dicoccum (l'Amidonnier), Triticum aestivum/durum (Froment/blé) (Ballouche et Marinval 2003). A Haua Fteah (Cyrénaïque) des carporestes d'orge (Hordeum vulgare) et de blé (Triticum durum) domestiques, ont été recueillis en contextes libyco-capsiens, mais ne sont pas encore datés (Baker 2002, Barker et al. 2010). Des recherches se poursuivent au Mali et au Soudan (Neumann 2004, Neumann et al. 2009) dans un autre contexte agro-climatique et culturel (Pennisetum).

Dans les abris et grottes de l'Algérie atlasique et tellienne, aucune céréale domestique n'a été identifiée. Les pollens et carporestes permettent de détecter un radoucissement climatique au milieu de l'Holocène, marqué par le remplacement du frêne dimorpha, par le $F$. xanthoxyloides et l'abondance des fruits (raisin et glands), dans l'Aurès d'après $R$. Portères (in Roubet 1979). Ces faits de préhension évoquent la présence de ces arbres, mais plus encore celles de goûts, attestant le maintien des pasteurs en altitude jusqu'à maturation tardive des fruits. Les collectes réitérées de fruits témoignent d'une connaissance du rythme des fructifications échelonnées. Le cas de la glandée automnale est le plus typique (cf. N45 « Néolithisation atlasique »). Ces comportements récurrents traduisent un accroissement des connaissances sur les nouvelles potentialités nutritives de certains végétaux (farines, semoules de glands doux), ayant déclenché une exploitation rationnalisée. Traits liés à une gestion intégrée des collectes et de l'élevage. L'agriculture reste un sujet à l'étude. 


\section{Nouvelles perspectives}

Depuis quelques décennies des études de génétique des populations actuelles, de paléogénétique et de biochimie isotopique soutiennent la démarche transdisciplinaire adoptée pour analyser la néolithisation. Dans le domaine faunique (Vigne 2007, 2009, 2011), la mise en évidence de caractères discriminants contribue à préciser des voies de migrations de certains animaux domestiques depuis leurs foyers d'origine durant l'Holocène, tandis que d'autres chercheurs essaient de préciser les caractéristiques des régimes alimentaires. Dans le domaine botanique, la génétique moléculaire et la génomique contribuent à identifier plusieurs gênes clé de la domestication des céréales. Des résultats préliminaires montrent que quelques gênes auraient été la cible de la sélection anthropique et que leurs mutations auraient causé des changements phénotypiques majeurs permettant le passage de l'état sauvage à l'état cultivé. Grâce à la reconnaissance de nouveaux marqueurs certains généticiens tentent d'élucider l'origine de la domestication des plantes (Zeder 2008). Ces voies de recherches seront à développer en Afrique du Nord pour comprendre l'impact des mouvements fondateurs des sociétés historiques.

\section{Sphère symbolique}

Le développement de la sphère symbolique que l'on entrevoit de façon complexe et reliée à l'Homme moderne en Afrique, est un sujet réactualisé ici. Ne dissociant pas les phénomènes symboliques néolithiques de ceux qui les précédèrent, nous rappellerons brièvement leur nature, leur chronologie et les contextes qui les renfermaient.

$\mathrm{Au}$ Maroc oriental plusieurs coquilles marines intentionnellement perforées et ocrées (Nassarius gibbosulus*, ocre, gravure) ont été découvertes en contexte Atérien à Taforalt* (Bouzouggar et al. 2007 ; d'Errico 2003, d'Errico et al. 2006, 2009), datées de 82000 BP environ. Elles représentent le plus sûr ancrage d'un continuum symbolique, pérennisé jusqu'à l'Actuel (cf. Vanhaeren \& d'Errico, N14).

En Algérie orientale et littorale, le traitement précoce de l'argile a été mis en évidence par S. Hachi dans l'Ibéromaurusien (Pléistocène supérieur), entre 20 000-11 000 BP. Ce fait représente un nouvel ancrage comportemental - symbolique et technique-, inscrit à l'intérieur d'une nécropole*, haut-lieu symbolique aussi. Deux grottes, l'une à Tamar Hat (Saxon 1974, 1976), l'autre à Afalou Bou Rhummel (Hachi 1999, 2003a et b, 2006 ; Hachi et al. 2002), ont livré plusieurs figurines en argile modelée et cuite. Certaines, aux décors imprimés avant cuisson, montrent de nouvelles préoccupations esthétiques, conservées grâce à la maîtrise du feu. Cependant ces créations en argile modelée et cuite n'annoncent pas la préparation d'ustensiles domestiques céramiques.

Que sait-on de l'apparition de récipients céramiques? Au Sahara algérien l'usage de récipients céramiques a fait précocement son entrée au début de l'Holocène, sans qu'aucune connexion ne s'établisse avec l'évènement symbolique méditerranéen, cité ci-dessus. Ainsi, dans l'Atakor, le village néolithique du site Launey, fouillé par J.-P. Maître (1971) a livré quelques tessons céramiques décorés. Les deux datations sur charbons, établies à la fin des années soixante, sont les suivantes :

$9210 \pm 115$ BP soit 10189-10686 cal BP ou 8400 cal BC

$8475 \pm 100$ BP soit $09146-09680 \mathrm{cal} \mathrm{BP}$ ou $7350 \mathrm{cal} \mathrm{BC}$. 
Estimées trop anciennes lors de leur publication en 1968, ces datations furent écartées sans soupçonner un usage non domestique. En effet, une fonction symbolique attribuable à un récipient céramique préhistorique n'était pas encore une hypothèse envisagée. La marginalisation de cette documentation bloqua la réflexion. Or, voilà qu'ont été signalés au Niger et au Mali les plus anciens récipients céramiques connus à ce jour, suggérant d'autres informations qui réhabilitent ceux de l'Atakor en contribuant même à soutenir l'hypothèse d'un foyer originel saharien de production céramique. S'appuyant sur la présence des premier vases découverts au Mali (site de La Mouche : Huysecom et al. 2009) et au Niger, en contexte pré-pastoral, à Temet, Tamaya Mellet et Tagalagal (Roset 2000), A. Close (1995) et F. Jesse (2010) admettent que ces récipients, ornés de décors comparables, pourraient relever d'une production spécifique, répondant aussi à une fonction symbolique. Ces céramiques sont datées de :

$9370 \pm 130$ BP soit $10600 \pm 150$ cal BP ou $8650 \pm 150 \mathrm{cal} \mathrm{BC}$

$9330 \pm 130$ BP soit $10550 \pm 150$ cal BP ou $8600 \pm 150$ cal BC

41 Enfin, en prenant en compte d'autres documents, on a vu s'accroître en territoire septentrional, durant la néolithisation, la documentation symbolique (Jesse 2010, p. 221). Si, le domaine funéraire et celui de l'art rupestre atlasique - non daté fournissent diverses informations comportementales, aucune ne laisse entrevoir, pour l'instant, le cadre d'une vie sociale. Le bétail domestique gravé et peint (ovin, chien, bovin rarement) s'inscrit au cœur d'un bestiaire sauvage (carnivores) et d'herbivores exigeant de l'eau et de verts pâturages.

Dans les régions montagneuses de l'Algérie saharienne (Téfedest, Tassili), le domaine de l'art rupestre installe avec raffinement et détail d'inoubliables moments d'une vie sociale opulente. Les troupeaux abondants et diversifiés (bovins ovins, caprins, chiens) ne souffrent pas du manque de pâturage, ni de péjoration climatique. Des transhumances ont été déduites de la présence de rondes-bosses* d'animaux domestiques (ovins bovins) dans des campements découverts en plaines. On pourrait installer de tels témoignages durant les épisodes humides de l'Holocène ancien. Ces questions exigent des travaux multidisciplinaires.

\section{Conclusion}

43 Les étapes de la néolithisation en Afrique septentrionale ne se sont nulle part mises en place en même temps, ni de façon uniforme. Nous aurons à tenir compte de l'impact des connexions continentales avec l'Europe du sud, encore insuffisamment établi, avec l'Ibérie (Ramos et al. 2008) et l'Italie, prolongée par un chapelet d'îles voisines, mais aussi à nous appuyer sur les reliefs, traits structuraux majeurs, constituant des foyers attractifs, reliant entre eux, mais isolant aussi, les territoires méridionaux et tropicaux du Sahara, de la Mauritanie au Proche-Orient.

$\mathrm{Ne}$ ressemblant à aucun phénomène majeur antérieur, la Néolithisation est un processus sociétal fécond, dynamique, complexe, à approcher sans frontières, en développant les recherches sur la domestication animale et végétale dans leurs cadres environnementaux.

L'état actuel des connaissances sur la néolithisation nord-africaine durant l'Holocène est insuffisant. Les données de la subsistance montrent que le pastoralisme ne dût pas partout précéder l'agriculture, à l'exception peut être du Maroc rifain. L'élevage a 
assuré le développement et la diffusion des troupeaux en zones montagneuses. Trois grands ensembles pastoraux s'individualiseraient, provisoirement: saharien, tellien (comprenant deux ensembles distincts: à l'ouest, à céramique cardiales/à l'est, à impressa), et atlasique, développés au sein de contextes culturels différents.

D'autre part, accompagnant la maîtrise et le développement de l'élevage ovin, caprin, bovin, une région, l'Aurès, préfigure à travers ses occupations pastorales bimillénaires au milieu de l'Holocène, la mise en place par essaimage de petites structures communautaires pastorales autonomes, dynamiques, créatrices d'un ample mouvement de diffusion pastorale atlasique et d'une diversité socio-économique enracinée, transmise aux époques postérieures.

Toutes ces connaissances sont à accroitre pour donner une vision plus large de la néolithisation incluant d'autres conquêtes capitales: l'agriculture et ses techniques (céréales, arbres fruitiers), l'arrivée du cheval, des armes, qui représenteront à l'avenir des potentialités économiques, fondatrices d'une autochtonie et d'un épanouissement agro-pastoral berbères.

\section{BIBLIOGRAPHIE}

ALLEY R.B., MAYEWSKI P.A., SOWERS T.K., STUIVER M., TAYLOR K.C. \& CLARK P.U., 1997 - "Holocene climatic instability : a prominent widespread event 8200 yr ago", Geology, 25/6, p. 483-486. AUMASSIP G., 2001 - L'Algérie des premiers hommes, Paris, Maison des Sciences de l'Homme. BALLOUCHE A., 2001 - « Les paysages végétaux holocènes du Maghreb. Entre nature et culture », Actes des premières journées nationales d'archéologie, Rabat, vol. I, p. 120-130.

BALLOUCHE A., et MARINVAL P., 2003 - « Données palynologiques et carpologiques sur la domestication des plantes et l'agriculture dans le Néolithique ancien du Maroc septentrional (site de Kaf Taht el-Ghar) ", Revue d'archéométrie, t. 27, p. 49-54.

BARICH B., 2010 - Antica Africa. Alle origini delle società, Roma, L'Erma di Bretschneider.

BARKER G., 2002-“Transition to farming and pastoralism in North Africa”, in Examining the farming, language dispersal hypothesis, in Bellwood P. \& Renfrew C., (eds), Cambridge : Mac Donald Institute for Archaeological Research, Oxbow Book, p. 151-162.

BARKER G., HUNT., REYNOLDS T., BROOKS I., EL RISHI H., 2007 - “The Haua Fteah, Cyrenaica (Northeast Libya) : Renewed investigations of the cave and its landscape", Libyan Studies, t. 38, p. 93-114.

BARKER G., ANTONIADOU A., ARMITAGE S., BROOKS I., CANDY L, CONNELLL K., DOUKA K., DRAKE N., FARR L., HILL E., HUNT C., INGLIS R., JONES S., LANE C., LUCARINI G., MENEELY J., MORALES J., MUTRI G., PRENDERGAST A., RABETT R., READE H., REYNOLDS T., RUSSEL N., SIMPSON D., SMITH B., STIMPSON C., TWATI M. \& WHITE K. 2010 - "The Cyrenaican Prehistory Project 2010 : the fourth season of investigations of the Haua Fteah cave and its landscape, and further results from the 2007-2009 fieldwork", Libyan Studies, vol. 41, p. 63-88. 
BENSLAMA M., ANDRIEU-PONEL V., GUITER F., REILLE M., de BEAULIEU J.-L, MIGLIORE J., DJAMALI M., 2010 « Nouvelle contribution à l'histoire de la végétation en Algérie : analyse pollinique de deux profils sédimentaires de complexe humide d'El Kala », Ac. des Sc., Comptes Rendus Biologies, vol. 333, p. 744-754.

BERGER J.-F., GUILAINE J., 2009 - “The 8200 cal BP abrupt environmental change and the Neolithic transition : a Mediterranean perspective", Quaternary International, vol. 200, p. 31-49.

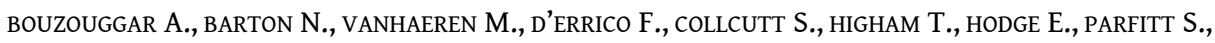
RHODES E., SCHENNIGER J.-L., STRINGER C., TURNER E., WARD S., MOUTMIR A. and STAMBOULI A., 2007 "82,000-year-old shell beads from North Africa and implications for the origins of modem human behaviour", Proceedings of the National Academy of Sciences, vol. 104, p. 9964-9969.

CALLOT Y., FONTUGNE M., 2008 - « Les sites lacustres d'âge holocène dans l'est du Grand Erg Occidental (nord-ouest du Sahara algérien) : interprétation géomorphologique et paléoclimatique ", Géomorphologie : relief, processus, environnement, nº 3, p. 187-200.

CLOSE A.E., 1995 - "Few and far between. Early ceramics in North Africa", in Barnett W.K. (ed), The emergence of pottery : technology and innovation in ancient society ; Washington DC, Smithonian Institution Press, p. 23-37,.

CREMASCHI M. et DI LERNA S. (eds), 1998 - Wadi Teshuinat. Palaeoenvironment and Prehistory in South Western Fezzan (Libyan Sahara). Quaderni di geodinamica alpine e del Quaternario, $n^{\circ} 7$, Milano.

CREMASCHI M. \& DI LERNA S., 1999 - "Holocene climatic changes and cultural dynamics in the Libyan Sahara ", African Archaeological Review, 16/4, p. 211-238.

DAUGAS J.-P., EL IDRISSI A., BALLOUCHE A., MARINVAL P., OUCHAOU B., 2008 - « Le Néolithique ancien au Maroc septentrional : données documentaires, sériation typo-chronologique et hypothèses génétiques », Bulletin de la Société Préhistorique Française, t. 105, p. 787-812.

DI LERNIA S. \& GARCEA E.A.A., 1997 - "Some remarks on Saharan terminology. Pre-pastoral archaeology from the Libyan Sahara and the Middle Nile valley", Libya Antiqua, n.s. 3, p. 11-23. D'ERRICO F. et al., 2009 - "Additional evidence on the use of personal ornaments in the Middle Palaeolithic of North Africa", Proceeding of National Academy of Science, mars,

D'ERRICO F., VANHAEREN M. et al., 2006 - "Middle Palaeolithic shell beads in Israël and Algeria", Science, 312, p. 1785-1788.

D'ERRICO F., 2003 - “An invisible frontier. A multiple species model for the origin of behavioral modernity", Evol. Anthropol., 12, p. 188-202.

EL IDRISSI M., 2001 - Le Néolithique ancien du Maroc septentrional dans son contexte régional, Thèse présentée à l'Institut National des Sciences de l'Archéologie et du Patrimoine, Rabat, non publiée. GARCEA E.A.A., 2004 - "An Alternative Way Towards Food Production : The Perspective from the Libyan Sahara", Journal of World Prehistory, vol. 18/2, p. 107-154.

GASSE F., FONTES J.-Ch.., PLAZIAT J.-C., CARBONNEL P., KACZMARSKA., de DEKKER P., SOULIE-MARSCHE, CALLOT Y., DUPEUBLE A., 1987 - "Biological remains, geochemistry and stable isotope for the reconstruction of environmental and hydrological changes, in the Holocene lakes from North Sahara", Palaeogeography, Palaeoclimatology, Palaeoecology, 60, p. 1-46.

GAST M., 1968 - Alimentation des populations de l'Ahaggar. Etude éthnographique, Paris, AMG (Mémoire VIII du CRAPE, Alger). 
GAUTIER A.,1984 - “Archaeozoology of the Bir Kiseiba region, Eastern Sahara”, in Cattle-keepers of the Eastern Sahara, Wendorf F. \& Schild R. (assemblers), Close A.E. (eds), Dallas, Department of Anthropology, Institute for the study of Earth and Man, Southern Methodist University, p. 49-72.

GAUTIER A., 1987 - "The archaeozoological sequence of the Acacus", In Archaeology and Environment in the Libyan Sahara. The excavations in the Tadrart Acacus 1978-1983, Barich B.E., (ed.), Oxford British Archaeological Reports, International Series, $n^{\circ} 368$. p. 283-308.

HACHI S., 1999 - L'homme de Mechta-Afalou, Cro-Magnon de l'Afrique du Nord, durant les 20 derniers millénaires : évolution culturelle et devenir, Thèse de HDR, Université Paul Valéry III, Montpellier, 4 volumes.

HACHI S., 2003a - Les cultures de l'Homme de Mechta-Afalou, Alger, CNRPAH (Mémoires, n.s. : 2).

HACHI S., 2003b - Aux origines des Arts Premiers en Afique du Nord. Les figurines et les objets modelés en terre cuite de l'Abri sous-roche préhistorique d'Afalou, Babors, Algérie (18 000-11000 BP), Alger, CNRPAH (Mémoires, n.s. : 6).

HACHI S., 2006 - « Du comportement symbolique des derniers chasseurs MechtaAfalou d'Afrique du Nord », C.R. Palevol, t. 5, fasc. 1-2, p. 429-440.

HACHI S., FRÖHLICH F., GENDRON-BADOU A., de LUMLEY H., ROUBET C., ABDESSADOK S., 2002 - « Figurines du Paléolithique supérieur en matière minérale plastique cuite d'Afalou Bou Rhummel (Babors, Algérie). Premières analyses par spectroscopie d'absorption infrarouge ", L'Anthropologie, t. 196, p. 57-97.

HIGGS, E. S., 1967 - "Environment and Chronology : the evidence from the mammal fauna", Chapter 2 in Mc Burney, C.B.M. 1967, The Haua Fteah (Cyrenaica) and the Stone Age of the South-East Mediterranean. Cambridge, Cambridge University Press, p. 16-44.

HUNT C.O., REYNOLDS T.G., EL-RISHI H.A., BUZALAN A., HILL E. \& BARKER G.W., 2011 - "Resource pressure and environmental change on the North African littoral : Epipalaeolithic to Roman gastropods from Cyrenaica, Libya", Quaternary International (sous presse).

HUTTERER. MIKDAD A., RIPKEN Th. E.J., 2011 - "Species composition and human exploitation of terrestrial gastropods from Taghit Haddouch, an early Holocene archaeological site in NE Morocco", Arch. Molluskenkunde (Frankfurtam-Main), 140, p. 57-75.

HUYSECOM E., 1996 - « Le concept de « Néolithique » en Afrique noire vu au travers d'observations ethnoarchéologiques ", in The Prehistory of Africa, XIII ${ }^{\text {th }}$ UISPP Congress, Forli, ABACO edizioni, p. 257-262.

HUYSECOM E. 2006 - « Vers un « Néolithique africain » : concept et apparition », Etudes maliennes, vol. 65, p. 43-67.

HUYSECOM E., RASSE M., LESPEZ L., NEUMANN K., FAHMY A., BALlOUCHE A., OZAINNE S., MAGGETTI M., TRIBOlO C. \& SORIANO S., 2009 - "The emergence of pottery in Africa during the $10^{\text {th }}$ millennium calBC : new evidence from Ounjougou (Mali)", Antiquity, 83 (322), p. 905-917.

JACKES M., et LUBELL D., 2008 - "Early and Middle Holocene Environments and Capsian Cultural Change : Evidence from the Télidjène Basin, Eastern Algeria", Afr. Archaeol. Rev., 25, p. 41-55.

JESSE F., 2010 - "Early pottery in Northern Africa. An overview", Journal of African Archaeology, vol. 8/2, p. 219-238.

LE QUELLEC J.-L. 2011 - "Provoking lactation by the insufflation technique as documented by the rock images of the Sahara", Anthropozoologica, 46.1, p 65-125. 
MALEY J., 1981 - Etudes palynologiques dans le basin du Tchad et paléoclimatologie de l'Afrique nordtropicale de 30000 ans à l'époque actuelle, Th. Sc. Montpellier, Trav. et Documents, Paris, $\mathrm{n}^{\circ} 129$, $586 \mathrm{p}$.

MALEY J., 2010 - "Climate and palaeoenvironment evolution of the North tropical Africa from the end of the Tertiary to the upper Quaternary", Paleoecology of Africa, vol. 30, p. 227-278.

MAITRE J.-P., 1971 - Contribution à la Préhistoire de l'Ahaggar 1. (Téfedest centrale), Paris, AMG (Mémoire XVII du CRAPE).

NEUMANN K., 2004 - "The romance of farming. Plant cultivation and domestication", in A.B. Stahl (ed.), African Archaeology : a critical introduction, Oxford, Blackwell, p. 249-275.

NEUMANN K., FAHMY A., LESPEZ L., BALLOUCHE A., HUYSECOM E., 2009 - “The Early Holocene palaeoenvironment of Ounjougou (Mali) : Phytoliths in a multiproxy context", Palaeogeography, Palaeoclimatology, Palaeoecology, 276, p. 87-106.

PANAUD O., 2009 - "The molecular bases of cereal domestication and the history of rice", C.R. Biologies, 332, p. 267-272.

PETIT-MAIRE N., et al. (ed.), 1979 - Le Sahara atlantique à l'Holocène, Paris, AMG (Mémoire XXVIII du CRAPE).

RAHMANI N., 2004. - "Technological and cultural change among the last huntergatherers of the Maghreb : the Capsian (10,000-6,000 BP)", Journal of World Prehistory, vol. 18, p. 57-105.

RAMOS J., ZOUAK M., BERNAL D., RAÏsSOUNI B., (ed.), 2008 - Las ocupaciones humanas de la cueva de Caf Taht el Ghar (Tetuan), Cadiz, Universidad de Cadiz.

REIMER M. et al, 2004. - "Intcal04 terrestrial radiocarbon age calibration, 0-26 cal KYR BP”, Radiocarbon, vol. 46/3, p. 1029-1058.

ROSET J.-P., 2000 - « Céramique et néolithisation en Afrique saharienne », in J. Guilaine (dir.), Premiers paysans du monde : naissance des agricultures, Paris, Editions Errance, p. 263-290.

ROUBET C., HACHI S., KHERBOUCHE F., 2011 - « Vivre ensemble depuis 20000 ans au moins en Afrique du Nord : expression d'une communication ", Nouveaux Mélanges offerts à Paulette Galand-Pernet et Lionel Galand, à l'occasion de leur 90e anniversaire, A. Mettouchi éd., Cologne, Rüdiger Köppe Verlag, p. 1-30.

SALAMANI M., 1991 - « Premières données palynologiques sur l'histoire Holocène du massif de l'Akfadou (Grande Kabylie, Algérie) », Ecologia Mediterranea, t. XVII, p. 145-159.

SAXON E. C., CLOSE A., CLUZEL Ch., MORSE V., CHACKLETON N.-J., 1974 - "Results of recent investigations at Tamar Hat", Libyca, XXII, p. 49-82.

SAXON E. C., 1976 - Prehistoric economies of the Israel and Algerian littorals : 18 000-8000 BP. Cambridge University, Jesus College, $233 \mathrm{p}$.

VANHAEREN M. et al., 2006 - "Middle Palaeolithic shell beads in Israel and Algeria", Science, vol. 312, p. $1785-1788$.

VIGNE J.-D., 2007 - « Exploitation des animaux et néolithisation en Méditerranée nordoccidentale », in Guilaine J., Manen C., Vigne J.-D. (dir.), Pont de Roque-Haute. Nouveaux regards sur la néolithisation de la France méditerranéenne, Toulouse, Archives d'Écologie Préhistorique, p. 221-301. 
VIGNE J.-D., 2009 - «La paléogénétique en paléontologie, archéologie et paléoanthropologie : apports et limites », C.R. Palevol, 7, p. 81-90.

VIGNE J.-D., 2011 - "The origins of animal domestication and husbandry : a major change in the history of humanity and the biosphere", C.R. Biologie, vol. 334, p. 171-181.

WENINGER B., ALRAM-STERN E., BAUER E., CLARKE I., DANZEGLOCKE U., JÖRIS O., KUBATZKI C., ROLLEFSON G., TODORVA H., TJEERD VAN ANDEL, 2006 - "Climate forcing due to the $8200 \mathrm{Cal}$ BP event observed at early Neolithic sites in the Eastern Mediterranean”, Quaternary Research, 66, p. 401-420.

WENDORF F., SCHILD R. \& CLOSE A.-E., 1984 - Cattle-keepers of the Eastern Egyptian Sahara. Dallas, Department of Anthropology, Institute for the study of Earth and Man, Southern Methodist University.

WENDORF F., SCHILD R. \& Associates, 2001 - Holocene settlement of the Egyptian Sahara vol. 1 : The archaeology of Nabta Playa, New York, Kluwer Academic, Plenum Publishers.

WENGLER L., DELIBRIAS G., EVIN J., et FONTUGNE M., 1999 - « Datations par le Radiocarbone des cultures préhistoriques en relation avec l'environnement dans l'Est du Maroc », in Evin J., Oberlin C., Daugas J.-P., Salles J.-F., eds. 14C and l'Archéologie. 3e Congrès International, Lyon, 6-10 avril 1998, Mém. de la SPF : 26, p. 371-439.

ZEDER M.-A., 2008 - "Domestication and early agriculture in the Mediterranean Basin : Origins, diffusion, and impact”, Proceeding of National Academy of Science, vol. 105, n 33, p. 11597-11604.

ZIELHOFFER C. \& LINSTÂDTER J., 2006 - "Short-term mid-Holocene climatic deterioration in the West Mediterranean region : climatic impact on Neolithic settlement pattern", Zeitschrift für Geomorphologie, vol. 142, p. 1-17.

ZIELHOFFER C., FAUST D., LINSTÄDTER J., 2008 - "Late Pleistocene and Holocene alluvial archives in the Southwestern Mediterranean : Changes in fluvial dynamics and past human response", Quaternary International, vol. 181, p. 39-54.

\section{INDEX}

Mots-clés : Art rupestre, Néolithique 